\title{
Necessidades Atuais de Radioterapia no SUS e Estimativas para o Ano de 2030
}

Current Radiotherapy Needs in SUS and Estimates for the Year 2030

Las Necesidades Actuales de Radioterapia en el SUS y las Estimaciones para el Año 2030

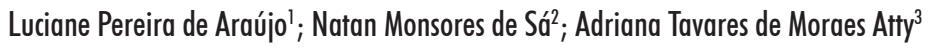

\section{Resumo}

Introduçáo: O câncer se encontra entre as principais doenças crônicas não transmissíveis do mundo e, para 2030, é esperado um aumento na incidência de $54 \%$ em relação a 2015. Esse incremento requer estratégias e políticas públicas que visem atender às demandas por tratamento, especialmente terapia de radiação, uma vez que envolve equipamentos e recursos humanos específicos. Objetivos: Apresentar o cenário atual da radioterapia no Brasil, seus recursos estruturais e humanos, bem como estimar o cenário da radioterapia para o país em 2030. Método: Trata-se de um estudo descritivo realizado com base em referencial teórico e conformado a partir de estimativas populacionais, atual e projetada, bancos de dados de sistemas de informação oficiais e sitios de classe profissional, e em legislação e normas emitidas por órgãos que normatizam e licenciam o tema no Brasil. Resultado: Os resultados obtidos demonstram que o déficit em julho de 2015 no Brasil é de 255 serviços de radioterapia e em recursos humanos de 387 radioterapeutas, 546 físico-médicos e 425 supervisores de produção. Para o ano de 2030, o déficit estimado é de 198 serviços de radioterapia e em recursos humanos de 235 físico-médicos e 114 supervisores de produção. Conclusão: $\mathrm{O}$ envelhecimento populacional e o consequente aumento das doenças crônicas não transmissíveis como o câncer exigem dos serviços públicos de saúde um planejamento que garanta as demandas por tratamento. O déficit, atual e projetado, de radioterapia no Brasil sinaliza a urgência de estratégias e políticas públicas capazes de atender às necessidades apresentadas.

Palavras-chave: Radioterapia/estatística \& dados numéricos; Radioterapia (Especialidade)/estatística \& dados numéricos; Estudos Prospectivos; Política de Saúde; Brasil

\footnotetext{
${ }^{1}$ Analista em C\&T do Ministério da Saúde (MS), Instituto Nacional de Câncer José Alencar Gomes da Silva (INCA). Rio de Janeiro (RJ), Brasil. Especialista em Inteligência de Futuro pela Universidade de Brasília (UnB) em parceria com a Fundação Oswaldo Cruz (Fiocruz) e Master of Business Administration em Gerenciamento de Projetos pela Universidade Federal Fluminense (UFF). E-mail: luciane.araujo@inca.gov.br.

${ }^{2}$ Professor-Adjunto da UnB. Doutor em Bioética pela UnB. Brasília (DF), Brasil.E-mail: monsores@unb.br.

${ }^{3}$ Tecnologista em C\&T do MS, INCA. Rio de Janeiro (RJ), Brasil. Mestre em Saúde Pública pela Fiocruz. E-mail: aatty@inca.gov.br.

Endereço para correspondência: Luciane Pereira de Araújo. Rua Marquês de Pombal, 125/7o andar - Centro. Rio de Janeiro (RJ), Brasil. CEP: 20.230-240. E-mail: luciane.araujo@inca.gov.br.
} 


\section{INTRODUÇÃO}

A aceleração no processo de transição demográfica e epidemiológica do Brasil, ocorrida em menos de 50 anos, conduziu o país a um perfil sanitário caracterizado por enfermidades crônicas complexas e onerosas, entre elas o câncer ${ }^{1}$.

Denomina-se câncer um conjunto de mais de 100 doenças que têm em comum o crescimento desordenado de células, considerado como maligno, e que podem eventualmente invadir tecidos e órgãos, espalhando-se para outras regiôes do corpo. Suas causas são multifatoriais podendo estar relacionadas ao meio ambiente, hábitos ou costumes próprios de um ambiente sociocultural ou, ainda, geneticamente pré-determinados, podendo também estar ambas as motivaçóes interrelacionadas ${ }^{1}$.

Segundo o Instituto Nacional de Câncer José Alencar Gomes da Silva (INCA)1, dados epidemiológicos sobre a doença no país indicam que a estimativa 2012/2013 foi de 518.510 casos novos de câncer no Brasil, sendo 257.870 em homens e 260.640 em mulheres, sendo sua ocorrência preponderante em faixas etárias acima dos 50 anos. Os dados apontam ainda que o câncer representa a segunda causa de óbito na população.

Uma projeção da International Agency for Research Cancer (IARC) indica que, no Brasil, em 2030, haverá 733.340 novos casos de câncer excetuando-se o de pele náo melanoma. Isso representa um aumento de aproximadamente $54 \%$ em comparação com $2015^{2}$.

A radioterapia é uma das modalidades de tratamento do câncer que pode ser empregada em conjunto com a quimioterapia, a cirurgia ou o transplante de medula óssea, a depender de critério médico. Calcula-se que aproximadamente $50 \%$ dos pacientes oncológicos devem receber terapia de radiaçáo, isoladamente ou como uma alternativa ou adjuvante para o tratamento cirúrgico ${ }^{3}$.

Segundo a IARC e a International Atomic Energy Agency (IAEA), instituiçôes internacionais técnico-científicas norteadoras mundiais do tema do câncer e da radioterapia, a estimativa para a execução de um planejamento de serviços de radioterapia, equipamentos e recursos humanos envolvidos, se baseia na incidência de casos novos de câncer por ano ${ }^{3,4}$. A atual parametrização utilizada no Brasil para o planejamento de estabelecimentos de saúde voltados à oncologia é preconizada pela Portaria MS/SAS $\mathrm{n}^{\circ} 140$, de 27 de fevereiro de 2014, a qual estabelece a base populacional como referência. Essa portaria admite ponderaçóes específicas nos seus parâmetros, no entanto essas ponderaçóes não são restritas ao perfil epidemiológico existente no país 5 .

O presente trabalho se propóe prospectivo e pretende abordar as questóes de recursos humanos e de infraestrutura que envolvem o tema no Sistema Único de Saúde (SUS), a fim de apresentar o cenário atual dos serviços de radioterapia, levantar a sua atual oferta de máo de obra em nível superior, identificar estratégias e políticas públicas em curso voltadas para a questáo e apontar a tendência de cenário 2030 para o Brasil, caso se mantenham as atuais condições de intervenção na área.

\section{MÉTODO}

O trabalho é um estudo descritivo realizado com base em referencial teórico das instituiçóes internacionais de cooperação científica, IARC e da IAEA, e construído a partir de levantamento de dados secundários, cujas fontes básicas são os sistemas de informação oficiais do Cadastro Nacional de Estabelecimentos de Saúde (CNES) e do Instituto Brasileiro de Geografia e Estatística (IBGE), além daquelas disponíveis em órgão licenciador (Comissão Nacional de Energia Nuclear/CNEN), conselho e associação de classe (Conselho Federal de Medicina/CFM e Associação Brasileira de Físicos Médicos/ABFM).

Os requisitos para determinação dos dados utilizados e definição da metodologia atenderam às exigências de documentos legais (portarias, normas, resoluçôes e regulamentos), emitidos por órgãos que normatizam o tema no Brasil (Agência Nacional de Vigilância Sanitária/ Anvisa e CNEN), revelados em pesquisa documental preliminar.

Inicialmente foram identificados os bancos de dados de sistemas de informação oficiais e sitios de classe profissional que continham informaçôes disponíveis necessárias à elucidação dos aspectos de construção da pesquisa. Desse modo, os passos que consolidaram essa etapa foram:

1. Coleta no sítio do IBGE da estimativa 2015 e da projeção da população do Brasil em 2030'.

2. Coleta no $\mathrm{CNES}^{7}$ de equipamentos de radioterapia existentes em hospitais habilitados, anexo V da Portaria MS/SAS no 140/2014 e atualizaçôes ${ }^{5,8-12}$.

3. Coleta do número de equipamentos de radioterapia a serem instalados em estabelecimentos de saúde habilitados ou a serem habilitados no SUS, por meio de programas de incentivo do Governo (Projeto Expande e Plano de Expansão da Radioterapia) ${ }^{13,14}$ e, em modalidades de financiamento federal, como os convênios e contratos de repasse cadastrados nos Sistemas de Pagamento (SISPAG), Sistema de Gestão de Convênios (SICONV) ou Sistema de Gestão Financeira e de Convênios (GESCON), considerando aqueles com término posterior à publicação da Portaria MS/SAS no 140/2014 e não contemplados pela mesma - obtidos por meio do Portal Transparência, opçáo convênios, em download de dados ${ }^{15}$. 
4. Coleta, em sitios de classe (CFM e ABFM) e do Governo (CNEN), de profissionais habilitados na forma exigida pela Portaria MS/SAS no 140/2014, pelas Resoluçóes CNEN no 130/2012 e no 166/ 2014 e pela Resolução (RDC) Anvisa no 20/2006, as quais normatizam, autorizam e licenciam os serviços de radioterapia no país ${ }^{16-21}$.

5. Coleta de vagas disponibilizadas por ano em residências médicas em radioterapia, em instituiçóes reconhecidas pelo Ministério da Educaçáo (MEC), segundo a Sociedade Brasileira de Radioterapia $(\mathrm{SBRT})^{22}$ e de vagas disponibilizadas por ano em cursos de especialização em física-médica ${ }^{23-25}$;

6. Coleta de vagas disponibilizadas, por meio do Programa Nacional de Apoio à Atenção Oncológica (PRONON), para incentivo à formação de recursos humanos (radioterapeutas e físico-médicos) ${ }^{26,27}$.

A análise dos dados consistiu em mensurar as relaçôes entre as informaçóes coletadas e as determinaçóes legais impostas à operação de serviços de radioterapia no país, comparando os dados observados com os dados teoricamente esperados pelas normativas, mensurando o distanciamento entre elas, bem como estimar as necessidades para o ano de 2030.

Torna-se relevante esclarecer que na descrição dos cenários atual e futuro foi utilizada como referência em planejamento de serviços de radioterapia a base populacional para a parametrizaçáo dos dados, conforme preconizado na Portaria MS/SAS no 140 , de 27 de fevereiro de 20145. A decisão de exclusão de ponderações específicas nesses respectivos cenários foi tomada uma vez que a portaria não exige, mas, apenas, admite a possibilidade de adoção de tais ponderaçóes.

\section{RESULTADOS}

\section{A) CENÁRIO ATUAL}

A construção do cenário atual teve por base a população estimada do Brasil, a qual alcançou em 2015 o total de 204.450.649 habitantes.

Considerando a razão de um estabelecimento de saúde para cada 500 mil habitantes, conforme preconizado na Portaria MS/SAS no 140 , de 27 de fevereiro de 2014, estima-se um total de 409 estabelecimentos de saúde necessários ao atendimento da demanda da populaçáo total brasileira para o ano de $2015^{5}$.

Ainda, segundo a portaria, o estabelecimento de saúde habilitado como Centro de Assistência de Alta Complexidade em Oncologia (Cacon) ou Unidade de Assistência de Alta Complexidade em Oncologia (Unacon) deverá observar o parâmetro mínimo de produção anual, em radioterapia, de 43 mil campos, sendo o cálculo do número de procedimentos mencionado correspondente ao funcionamento de um equipamento instalado de radioterapia externa de megavoltagem (teleterapia). Outrossim, a norma exige, ainda, que a Unacon faça a referência formal para radioterapia de seus usuários.

Dessa forma, infere-se das prescrições normativas que cada Unacon ou Cacon deva possuir minimamente um equipamento de teleterapia (unidade de cobalto ou acelerador linear) ou, ao menos, na hipótese de sua inexistência na Unacon, que seja garantido por meio de contrarreferência à produção mínima exigida. Assim, resulta de tal condição a necessidade de 409 equipamentos, entre cobalto e acelerador linear, para atendimento à demanda imposta pela portaria.

Para obtenção do licenciamento e autorização de funcionamento dos serviços de radioterapia, devem ser respeitadas as normas vinculantes ao tema, as quais exigem recursos humanos mínimos. Com isso, e dado o número de equipamentos necessários para atender à população estimada para 2015, chega-se à quantidade mínima de profissionais de nível superior requerida pelas normativas vigentes, quais sejam: 818 radioterapeutas e 818 físico-médicos, considerando que são exigidos substitutos desses profissionais quanto à responsabilidade técnica dos serviços, e, ainda, a possibilidade de acumulação de função do físico-médico como supervisor de radioproteção.

Segundo levantamento realizado para a presente pesquisa, o Brasil conta com 143 hospitais habilitados com serviço de radioterapia e 11 serviços isolados de radioterapia, totalizando 154 serviços de radioterapia e 247 equipamentos, quer seja unidade de cobalto ou acelerador linear.

Ao que se refere a recursos humanos, o país possui 431 radioterapeutas, 272 físico-médicos e 393 supervisores de radioproteçáo, sendo que do cruzamento de informaçóes entre físico-médico e supervisor de radioproteção encontram-se 245 profissionais que possuem, cumulativamente, as habilitaçôes de físico-médico e supervisor de radioproteção.

A partir da necessidade apresentada pela base populacional e o levantamento da infraestrutura e máos de obra existentes e habilitadas para a operacionalização de serviços de radioterapia, expóe-se análise final do cenário atual do país quanto à sua capacidade de prover a demanda estabelecida nas normas.

A partir dos dados coletados, identifica-se que o Brasil apresenta um déficit de 255 serviços de radioterapia, e, portanto, de 255 equipamentos de radioterapia. Encontra-se déficit também em número de profissionais habilitados, uma vez que seriam necessários minimamente 387 radioterapeutas, 546 físico-médicos e 425 supervisores de produção para atender à demanda do déficit de serviços 
de radioterapia. No cálculo dos profissionais, não foi considerada a escala reduzida, para servidores federais que operam com Raios X e substâncias radioativas, estabelecida na Lei no 1.234 , de 14 de novembro de $1950^{28}$, pois a obrigação imposta pela Resolução CNEN 166/14 ${ }^{20}$ exige profissionais substitutos, assim a compensação do número de profissionais fica estabelecida.

\section{B) ESTIMATIVA BRASIL 2030}

No desenho do cenário Brasil 2030, foi considerada a manutenção das normas atuais no futuro e, sendo assim, considerou-se como base do seu desenvolvimento a populaçáo projetada para o Brasil, a qual deve alcançar em 2030 o total de 223.126.917 habitantes ${ }^{6}$.

Considerando, a razão de um estabelecimento de saúde para cada 500 mil habitantes, conforme preconizado na Portaria MS/SAS no 140 , de 27 de fevereiro de $2014^{5}$, atingiu-se um total de 446 estabelecimentos de saúde necessários ao atendimento da demanda da população total brasileira estimada em 2030, ou seja, 37 serviços de radioterapia a mais do que o cenário atual já requer.

Mantendo-se tal projeção, a necessidade de equipamentos, entre cobalto e acelerador linear, para atendimento à demanda imposta pela portaria, será de 446 equipamentos ou 37 equipamentos a mais do que o já previsto para o cenário atual.

A partir desses números, espera-se que a quantidade mínima de profissionais de nível superior requerida seja de 892 radioterapeutas e 892 físico-médicos, mais especificamente 74 radioterapeutas, 74 físico-médicos e 74 supervisores de radioproteção, ou ainda, apenas, 74 físico-médicos, considerando-se a possibilidade de acumulação de função do físico-médico como supervisor de radioproteção. O quantitativo específico de profissionais habilitados reflete a diferença encontrada dessa projeção para o cenário atual, no qual se ponderaram as consideraçóes profissionais normativas já debatidas no cenário anterior, tal como compensação de cálculo da redução de carga horária por profissional substituto.

Segundo informaçóes levantadas para o presente trabalho, encontram-se, em andamento, alguns incentivos de criação e/ou ampliaçấo de serviços de radioterapia no país, são eles, o Projeto de Expansão da Assistência Oncológica (Projeto Expande), implantado em 2000 pelo Ministério da Saúde (MS), com a coordenação do INCA ${ }^{13}$, com seis projetos em andamento; o Plano de Expansão da Radioterapia do Ministério da Saúde ${ }^{14}$, totalizando 80 soluções de radioterapia, e, por fim; modalidades de financiamento federal, por meio de convênios e contratos de repasse, realizados a partir do cadastro de propostas nos seguintes sistemas: SISPAG, SICONV ou GESCON, nos quais foram identificados, como em andamento, oito propostas para serviços de radioterapia.

Com isso, haveria um acréscimo iminente de 94 serviços de radioterapia, em relação ao atual cenário, o que reduziria o déficit total para 198 estabelecimentos de saúde e equipamentos, no período de 15 anos, entre o cenário em julho de 2015 e o cenário Brasil 2030.

No aspecto de avaliação de projeção para recursos humanos, foi considerado o número de vagas disponíveis por ano para residência médica em radioterapia, no total de 61, o que permite vislumbrar uma dimensão de 305 radioterapeutas formados em um prazo de 15 anos, considerando o prazo de formação de três anos dessa residência médica, o que reduziria, mas manteria a existência de déficit de 156 profissionais. Quanto à projeção de físico-médicos, os dados coletados não são conclusivos, pois as informaçóes encontradas de cursos habilitados pelo MEC e de vagas estimadas, nas referências bibliográficas, estão desatualizadas ou díspares, entre si.

No entanto, o PRONON, criado pelo Governo Federal ${ }^{26}$, que, entre outros, visa à formação, ao treinamento e ao aperfeiçoamento de profissionais em radioterapia, deferiu vários projetos, mediante incentivo fiscal, para a formação de recursos humanos para suprir a demanda do SUS em sintonia com o Programa Nacional de Radioterapia do Ministério da Saúde ${ }^{14}$, o qual corresponde ao Plano de Expansão do próprio MS. Considerando essa informação, o déficit de radioterapeutas deixaria de existir, no entanto, ainda haveria um déficit de físico-médicos, pois ainda que se considere um total de 160 físico-médicos e de 160 supervisores de radioproteção habilitados a partir do PRONON, se teria ainda uma necessidade em torno de 460 físico-médicos e 339 supervisores de radioproteção, considerando-se déficit atual e projetado.

A média encontrada, entre os dados obtidos, de 15 físico-médicos formados por ano, em um período de 15 anos, não supriria a necessidade, a qual ainda seria de 235 físico-médicos e de 114 supervisores de radioproteção. Alerta-se, ainda, que a previsão da formação em física médica náo garantiria o atendimento do déficit, uma vez que esses profissionais ainda necessitariam obter habilitaçáo por meio de sua entidade classista (ABFM) e certificação do órgáo de licenciamento (CNEN).

\section{DISCUSSÃO}

Conforme apresentado nesta pesquisa, as informaçóes que se obtêm sobre o tema não são condensadas em único ambiente de consulta, nem são tão evidentes. Muitas vezes, os dados estáo desatualizados ou são desconexos, entre si, dificultando uma avaliaçáo concreta sobre o assunto. Tal problema já foi apontado pelo Tribunal 
de Contas da União (TCU ${ }^{29}$ em acórdáo que trata de auditoria operacional relativa à Política Nacional de Atenção Oncológica, no qual problemas com os dados dos sistemas de informação do SUS foram observados. Nele, o Tribunal critica a competência para alimentar e manter atualizados os dados, que é dos próprios estabelecimentos e das secretarias estaduais e municipais de saúde.

As inconsistências constatadas pelo TCU puderam ser sentidas no levantamento das Fichas dos Estabelecimentos de Saúde (FCES) no CNESNet, para a presente pesquisa, na qual foram detectados 15 estabelecimentos de saúde habilitados e identificados no Anexo V da Portaria MS/ SAS no 140 , de 27 de fevereiro de $2014^{5}$, com serviços de radioterapia, e para os quais simplesmente não havia informaçôes de radioterapia na FCES.

Ao que se refere a Recursos Humanos, nenhum centro de radioterapia deve ser operado sem pessoal qualificado, entre eles, radioterapeutas, físico-médicos de radioterapia, tecnólogos de radioterapia e outros, sendo assim, outro ponto relevante a ser exposto é a inexistência de regulamentação, no Brasil, da profissão de físico-médico ${ }^{30}$, o que levou a pesquisa a se apoiar, para análises relativas ao exercício da profissão, em normativas vigentes circundantes ao tema da radioterapia e na regulamentação da ABFM.

Pode ser observado que tal questão acabou por levar também a dificuldade de busca por informaçóes que apoiassem seguramente o estudo. O problema da inexistência de regulamentação da profissão de físico-médico também já foi apontado em outros trabalhos, nos quais também restaram demonstradas a necessidade e a importância de aprovação de Projeto de Lei que regulamente a profissão ${ }^{30}$.

Notou-se também uma grande superposição de normas vinculantes, que se não chegam a ser conflitantes, dificultam o levantamento e a avaliação dos dados, principalmente quanto ao que se refere o profissional de física médica e sua respectiva habilitação e exercício da profissão.

Ainda, quanto ao aspecto legal, observam-se a mudança dos parâmetros para o planejamento de estabelecimentos de saúde e suas respectivas habilitaçóes em oncologia impostas pela norma atual - base populacional - daqueles encontrados na portaria anterior, a qual tratava a questáo sob a dimensão de casos novos de câncer e de capacidade instalada - número de equipamentos - por macrorregiôes das unidades federativas. Torna-se esse ponto relevante, uma vez que as orientações das instituiçôes internacionais de cooperação científica também se baseiam em casos novos de câncer. Essa lógica se vê ainda refletida também em normas vigentes no país, inclusive as da CNEN. Não foram encontrados trabalhos que avaliassem e/ou abordassem o impacto de tal mudança quanto aos aspectos epidemiológicos e de cobertura assistencial no país. Tal questão merece destaque, pois um planejamento que se baseia em parâmetros de casos novos de câncer e de capacidade instalada configura uma nova lógica de cálculo de serviços de radioterapia, equipamentos e profissionais diversos daqueles aqui apresentados.

Finalmente, a pesquisa demonstrou que, apesar das açôes realizadas pelo Governo Federal, os programas executados, até a presente data, não são suficientes à superaçáo do déficit e demanda porvir dos casos de câncer previstos para o Brasil. Da mesma forma, artigo publicado no sítio da SBRT ${ }^{31}$ considerando o panorama da radioterapia trouxe análise similar. Ainda, nesse diapasão, Grover et al..$^{32}$ e Zubizarreta et al. ${ }^{33}$, em uma abordagem global, baseados em dados do Directory of Radiotherapy Centres (DIRAC), banco de dados do IAEA, também apontaram o déficit de serviços de radioterapia, equipamentos e máo de obra, inclusive no Brasil, enfatizando a capacitaçáo de recursos humanos como ponto crucial do planejamento e gestáo em radioterapia.

Sob esse aspecto, ressalta-se que o PRONON, muito embora seja um programa que, entre outros, incentiva açóes de capacitaçáo de Recursos Humanos em radioterapia, no país, não garante a realização dos projetos, uma vez que depende da captação de incentivo fiscal para sua realização. Assim, não há certeza da formação prevista de profissionais necessários, na área, para atender à demanda do Plano de Expansão da Radioterapia do Ministério da Saúde e de casos novos de câncer até o ano de 2030.

Por último, deve-se salientar que, no presente trabalho, os serviços de radioterapia oferecidos na saúde suplementar não foram considerados nos cálculos realizados, uma vez que o SUS se pretende universal e igualitário. Caso contrário, os resultados quantitativos apresentados seriam reduzidos, em média, 20\% dos totais alcançados ${ }^{34}$, alterando os cenários aqui conformados.

\section{CONCLUSÃO}

A avaliação do cenário atual da radioterapia no Brasil e a estimativa para 2030 trazem à tona informaçóes relevantes que permitem observar e revelar a situação dessa modalidade de tratamento, no SUS, bem como os problemas advindos do tema no país, uma vez que há de se garantir investimentos financeiros, tecnológicos e em recursos humanos que assegurem o pronto e imediato atendimento aos usuários do SUS.

As exigências inerentes à questáo da radioterapia, principalmente em Recursos Humanos, requerem estratégias e políticas públicas contínuas capazes de confrontar as demandas constantes geradas pelo aumento do número de casos novos de câncer no país. 
Os apontamentos apresentados nesse estudo demonstram a urgência de açóes que tornem confiáveis os sistemas oficiais de informação, que regulamentem a profissão de físico-médico e que atendam às necessidades requeridas pelos casos novos de câncer, a fim de que o impacto desses problemas seja refreado desde já.

\section{CONTRIBUIÇÕES}

Luciane Pereira de Araújo contribuiu na concepçáo, planejamento do projeto de pesquisa, redação, obtençáo e/ou análise e interpretaçáo dos dados. Natan Monsores de Sá contribuiu na concepção e planejamento do projeto de pesquisa, redação e revisão crítica. Adriana Tavares de Moraes Atty contribuiu na redação e revisão crítica, obtenção e/ou análise e interpretaçáo dos dados.

\section{DECLARAÇÃO DE CONFLITO DE INTERESSES}

O autor Natan Monsores de Sá declara não possuir conflito de interesse, mas as autoras Luciane Pereira de Araújo e Adriana Tavares de Moraes Atty declaram potencial conflito de interesse pela condição de possuírem vínculos empregatícios com o INCA.

\section{REFERÊNCIAS}

1. Instituto Nacional de Câncer José Alencar Gomes da Silva. $\mathrm{ABC}$ do câncer: abordagens básicas para o controle do câncer. 2. ed. Rio de Janeiro: Inca; 2012.

2. International Agency for Research on Cancer. GLOBOCAN 2012: Estimated cancer incidence mortality and prevalence worldwide in 2012: prediction [Internet]. Lyon, France: IARC; 2013 [acesso em 2015 fev. 4]. Disponível em: http://globocan.iarc.fr/Pages/ burden_sel.aspx.

3. International Atomic Energy Agency. Planning national radiotherapy services: a practical tool. Vienna: International Atomic Energy Agency; 2010.

4. International Atomic Energy Agency. Setting up a radiotherapy programme: clinical, medical physics, radiation protection and safety aspects. Vienna: International Atomic Energy Agency; 2008.

5. Brasil. Portaria MS/SAS no 140 , de 27 de fevereiro de 2014. Diário Oficial da União, Poder Executivo, Brasília, DF, 28 fev. 2014. Seção 1, n. 42, p. 71.

6. Instituto Brasileiro de Geografia e Estatística. Projeções e estimativas da população do Brasil e das unidades da federação [Internet]. [Rio de Janeiro]: IBGE; [acesso em 2015 jan. 23]. Disponível em: http://www.ibge.gov.br/ apps/populacao/projecao/index.html.

7. Cadastro Nacional de Estabelecimentos de Saúde [Internet]. Brasília, DF: Ministério da Saúde; [acesso em 2016 abr. 28]. Disponível em: http://cnes2.datasus.gov. br/Lista_Es_Nome.asp?VTipo=0,
8. Brasil. Portaria $\mathrm{n}^{\circ} 132$, de 24 de fevereiro de 2014. Diário Oficial da União, Pode Executivo, Brasília, DF, 25 fev. 2014. Seção 1, n. 39, p. 105.

9. Brasil. Portaria no 278, de 4 de abril de 2014. Diário Oficial da União, Poder Executivo, Brasília, DF, 07 abr. 2014. Seção 1, n. 66, p. 48.

10. Brasil. Portaria no 653, de 30 de julho de 2014. Diário Oficial da União, Poder Executivo, Brasília, DF, 31 jul. 2014. Seção 1, p. 100.

11. Brasil. Portaria no 680, de 6 de agosto de 2014. Diário Oficial da União, Poder Executivo, Brasília, DF, 12 ago. 2014. Seção 1, n. 153, p. 40.

12. Brasil. Portaria no 689, de 7 de agosto de 2014. Diário Oficial da União, Poder Executivo, Brasília, DF, 08 ago. 2014. Seção 1, p. 45.

13. Instituto Nacional de Câncer José Alencar Gomes da Silva. Expansão da assistência oncologia: projeto EXPANDE [Internet]. Rio de Janeiro: Inca; [acesso em 2015 jan. 16]. Disponível em: http://www2.inca.gov.br/ wps/wcm/connect/acoes_programas/site/home/nobrasil/ projeto_expande.

14. Brasil. Portaria no 931, de 10 de maio de 2012. Diário Oficial da União, Poder Executivo, Brasília, DF, 11 maio 2012. Seção 1, n. 91, p. 140.

15. Controladoria Geral da União (BR). Portal da Transparência: Downloads de dados: convênios [Internet]. Brasília, DF: CGU; [acesso em 2015 jan. 22]. Disponível em: http://www.portaltransparencia. gov.br/downloads/snapshot.asp?c=Convenios\#get.16. Conselho Federal de Medicina (BR). Busca de médicos [Internet]. Brasília, DF: CFM; [acesso em 2014 dez. 11]. Disponível em: http://portal.cfm.org.br/index.php?med icosNome $=\&$ medicosCRM $=\&$ medicosUF $=\&$ medicos $S$ ituacao $=A \&$ medicos TipoInscricao $=\&$ medicosEspecialid ade $=60 \&$ buscaEfetuada $=$ true\&option $=$ com_medicos.

17. Associação Brasileira de Física-Médica. Profissionais certificados: radioterapia [Internet]. Porto Alegre: ABFM; [acesso em 2014 dez. 24]. Disponível em: http://www.abfm.org.br/index.php?site=especialistas. php\&id=RT\&m=3. 18. Comissão Nacional de Energia Nuclear (BR). Supervisores de radioproteção: radioterapia [Internet]. Rio de Janeiro: CNEN; [acesso em 2014 dez. 10]. Disponível em: http://www.cnen.gov.br/seguranca/ cons-ent-prof/lst-prof-credenciados.asp?OP=FT.

19. Brasil. Resolução n ${ }^{\circ} 130$, de 31 de maio de 2012. Diário Oficial da União, Poder Executivo, Brasília, DF, 4 jun. 2012. Seção 1, p. 16.

20. Brasil. Resolução no 166 de 2014. Diário Oficial da União, Poder Executivo, Brasília, DF, 29 abr. 2014.

21. Ministério da Saúde (BR). RDC no 20, de 02 de fevereiro de 2006. Brasília, DF, 2003. Disponível em: http://portal. anvisa.gov.br/wps/wcm/connect/6d92c1804745975ca0 $00 \mathrm{f} 43 \mathrm{fbc} 4 \mathrm{c} 6735 / \mathrm{RDC}+\mathrm{N} \% \mathrm{C} 2 \% \mathrm{BA}+20$, +DE+02+DE+ FEVEREIRO+DE+2006. pdf?MOD=AJPERES $>$. 
22. Sociedade Brasileira de Radioterapia. Residências médicas reconhecidas pelo MEC [Internet]. São Paulo: SBR; 2015. [acesso em 2016 abr. 28]. Disponível em: http:// www.sbradioterapia.com.br/residencia-medica/servicosde-residencia/.

23. CHUERI. P. Política Nacional de Prevenção e Controle do Câncer na RASPDC e o Plano de expansão da Radioterapia. In: Workshop para soluções em formação de recursos humanos para radioterapia. Apresentação, 2014, Brasília, DF. Disponível em: http://portalsaude. saude.gov.br/images/pdf/2014/outubro/29/2-Apresenta---o-Patricia-Chueiri-SAS.pdf. Acesso em: 26 dez. 2014.

24. VIEGAS CMPV. Programa de expansão da radioterapia no Brasil formação de técnicos em radioterapia [Internet]. [Rio de Janeiro]: Inca; [acesso em 2014 dez. 26]. Disponível em: http://portalsaude.saude.gov.br/images/ pdf/2014/outubro/29/6-Aprtesenta----o-Celia-ViegasINCA.pdf.

25. Doreto, A. Rede de escolas técnicas do SUS/RET-SUS [Internet]. In: Workshop para soluções em formação de recursos humanos para radioterapia; 2014 out. 24; Brasília, DF. [acesso em 2014 dez. 26]. Disponível em: http:// portalsaude.saude.gov.br/images/pdf/2014/outubro/29/5Apresenta----o-Aldiney-Doreto-SGTES.pdf.

26. Brasil. Lei no 12.715 , de 17 de setembro de 2012. Diário Oficial [da] República Federativa do Brasil, Brasília, DF, 18 set. 2012 . Seção 1, n. 181, p.1.

27. Ministério da Saúde (BR). Programa Nacional de Apoio à Atenção Oncológica (PRONON) e Programa Nacional de Apoio à Atenção da Saúde da Pessoa com Deficiência (PRONAS/PCD) [Internet]. [Brasília, DF]: Ministério da Saúde; [acesso em 2015 abr. 13].

28. Brasil. Lei no 1.234, de 14 de novembro de 1950. Diário Oficial da União, Poder Executivo, Brasília, DF, 17 nov. 1950. Seção 1, p. 16553.
29. Tribunal de Contas da União. Acordão no AC-257738/14-P. Processo no 016.913/2013-2. Monitoramento. Auditoria operacional. Política Nacional de Atenção Oncológica. Algumas recomendações já implementadas. Expansão dos serviços de oncologia no SUS. Necessidade de novos esforços para a implementação das demais recomendaçôes. Autorização para o prosseguimento do monitoramento. Remessa de cópia aos órgãos interessados. Tribunal de Contas da União: São Paulo, 17 mar. 2015. Disponível em: https://contas.tcu.gov.br/ etcu/AcompanharProcesso?p1=16913\&p2=2013\&p3=2. Acesso em: 16 jan. 2015.

30. Ozório PJ. Considerações acerca da profissão do físico-médico: recomendaçōes, treinamento clínico e certificação [trabalho de conclusão de curso]. Santa Maria: Centro Universitário Franciscano; 2011.

31. Ferrigno R. Panorama da radioterapia no Brasil [Internet]. São Paulo: Sociedade Brasileira de Radioterapia; 2013 [acesso em 2015 fev. 4]. Disponível em: http://www. sbradioterapia.com.br/pdfs/panorama2013.pdf.

32. Grover S, Xu MJ, Yeager A, Rosman L, Groen RS, Chackungal S, et al. A systematic review of radiotherapy capacity in low- and middle-income countries. Front Oncol. 2015;4:380.

33. Zubizarreta EH, Fidarova E, Healy B, Rosenblatt E. Need for radiotherapy in low and middle income countries the silent crisis continues. Clin Oncol (R Coll Radiol). 2015;27(2):107-14.

34. Rede Interagencial de Informação para a Saúde. Indicadores básicos para a saúde no Brasil: conceitos e aplicações. 2. ed. Brasília, DF: Organização PanAmericana da Saúde; 2008. Capítulo F, Cobertura; p. 276-311. 


\begin{abstract}
Introduction: Cancer is amongst the main non-communicable chronic diseases in the world and for 2030 it is expected to increase in the incidence of $54 \%$ compared to 2015 . This increase requires public strategies and policies to meet the demands for treatment, especially therapy radiation, since it involves specific equipment and manpower. Objectives: To present the current scenario of radiotherapy in Brazil, its structural and human resources, and to evaluate the scene of radiation to the country in 2030. Method: This is a descriptive study based on reference theoretical and in current and projected population estimates, databases of official information systems and professional class, and legislation and regulations issued by agencies that regulate and license the subject in Brazil. Results: The results showed that the deficit in July 2015 in Brazil is 255 radiotherapy services and human resources of 387 radiation therapists, 546 physical-medical and 425 production supervisors. For the year 2030 the estimated deficit is 198 radiotherapy and human resources of 235 physical-medical and 114 production supervisor. Conclusion: The aging population and consequent increase in chronic diseases like cancer demand the public health services to do some planning to ensure the demand for treatment. The deficit, current and projected radiotherapy in Brazil indicates the urgency of strategies and public policies to meet the needs presented.

Key words: Radiotherapy/statistics \& numerical data; Radiation Oncology/statistics \& numerical data; Prospective Studies; Health Policy; Brazil
\end{abstract}

\title{
Resumen
}

Introducción: El cáncer es una de las principales enfermedades crónicas más no transmisibles del mundo y para el 2030 se espera un aumento en la incidencia del $54 \%$ en comparación con 2015. Este aumento requiere de estrategias y políticas públicas para satisfacer las demandas por tratamiento, especialmente la terapia la radiación, ya que se involucra equipos y mano de obra específica. Objetivos: Presentar el escenario actual de la radioterapia en Brasil, sus recursos estructurales y humanos, y para evaluar la escena de la radioterapia para el país en 2030. Método: Se trata de un estudio descriptivo con una base referencial teórica y conformado a partir de las estimativas poblacionales, actualizadas y proyectados, bases de datos de los sistemas de información y los sitios oficiales de clase profesional, y en emisiones de leyes y reglamentos de los organismos que regulan y autorizan el tema en Brasil. Resultados: Los resultados mostraron que el déficit en julio de 2015 en Brasil es de 255 servicios de radioterapia y recursos humanos de 387 radioterapeutas, 546 supervisores de producción-físicos médicos y 425. Para el año 2030 el déficit estimado es de 198 radioterapia y recursos humanos de los servicios 235 físicos médicos y 114 supervisores de producción. Conclusión: El envejecimiento de la población y el consiguiente aumento de las enfermedades crónicas como el cáncer exigen que los servicios de salud pública un planeamiento para asegurar las demandas del tratamiento. El déficit actual, en radioterapia es proyectado en Brasil indica la urgencia de estrategias y políticas públicas capaces de atender las necesidades que se presentan.

Palabras clave: Radioterapia/estadística \& datos numéricos; Oncología por Radiación/estadística \& datos numéricos; Estudios Prospectivos; Política de Salud; Brasil 